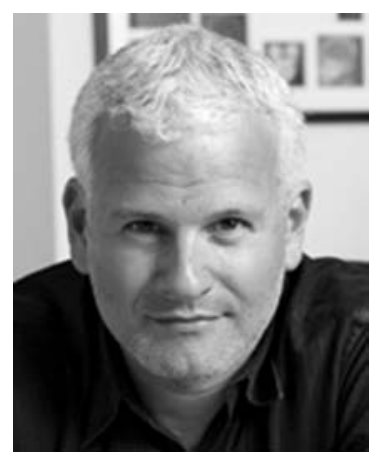

\title{
A Conversation with Adam Gazzaley
}

\author{
INTERVIEWER: ChARVy NARAIN
}

Former Senior Editor, Nature Neuroscience

Adam Gazzaley is the founding director of the Neuroscience Imaging Center at the University of California at San Francisco and a Professor in Neurology, Physiology, and Psychiatry.

Charvy Narain: You're studying how to improve the brain.

Dr. Gazzaley: Over the years we've done a lot of basic cognitive neuroscience research trying to understand how our brains function, how they are sensitive to interference, and how that gets worse as we get older. And then around 5 years ago, I started getting frustrated by having only shared the bad news that our brain function decays, without having anything we can do about it. We became driven to see if we could use the tools and the methodology that we have developed to understand the brain, and as a means of improving how the brain processes information.

Charvy Narain: What methods may people already have tried to ameliorate, say, the deficits associated with aging, or even just to improve normal functioning?

Dr. Gazzaley: There's a pretty broad landscape of approaches. The classic is education. That should be discussed because that's the mainstay of boosting how a brain operates. But other approaches have garnered some interest, such as pharmaceuticals. So drugs that are targeted at deficits associated with, let's say, Alzheimer's disease and work at the neurotransmitter system level have at least been considered as approaches to improve cognition. Then there's the general field of cognitive training. What we've been doing at our lab is related to that, perhaps with a little different spin on it.

Charvy Narain: Why should we consider cognitive training rather than popping a pill?

Dr. Gazzaley: In general, the pharmaceutical approach to enhancing how our brain functions has come up pretty short. None of the pharmaceuticals tried for the wide range of cognitive impairments in neurological and psychiatric diseases have worked very well. Some people are unaffected by them. Or they mostly get side effects, or a small effect, and it's generally because the pharmaceutical approach is a pretty blunt one. We can act on neurotransmitter systems, but we can't really act on circuits. So you wind up having to go to very high doses to get effects and then you get lots of side effects. There hasn't been a pill that really corrects these problems. The pharmaceutical approach is a sledgehammer, and the burden of side effects is just so great that the field has been reeling.

Charvy Narain: So what does cognitive training do? How does it work? And how do you actually do it?

Dr. Gazzaley: The drug approach, at least so far, can't activate a circuit. But interacting with a targeted environment - which might be in the form of a training program, in our case a video game - activates a circuit because that's how the brain works. Our idea is to activate that circuit and constantly challenge it by using adaptive algorithms, so that as you get better, the difficulty scales with you. The program keeps pushing on the system until it changes. In physical exercise, repeating a movement means the muscle gets stronger. So that's the general idea in cognitive training. It has selectivity because the interaction activates a circuit and because our brains are plastic, they modify themselves at many different levels in response to a challenge.

Charvy Narain: So what kind of circuits have you been able to get at?

Dr. Gazzaley: We dip back into our more basic science work in cognition and try to understand what systems are vulnerable and how we might design an interaction to really challenge that. In older populations, we know that there are deficits in cognitive control, such as selective attention, interference processing, multitasking, and distraction resistance. So our goals were to challenge prefrontal cortical circuits in older adults who were engaged in a task that had a lot of multitasking and distractions from other tasks.

Charvy Narain: What do these paradigms actually look like?

Dr. Gazzaley: One we've recently had success with and published last year was called NeuroRacer. The player is 
travelling through a 3D environment and trying to keep the car they're driving on the road, which is quite difficult, using a joystick so that it turns to the left, right, going up and down the hill. And while that's going on, signs are coming up and they have to respond as rapidly and accurately to target signs. So you have two tasks that are attentionally demanding occurring at the same time, and they both independently adapt. As you get better at them, they get faster and more difficult. Your rewards only occur when they both get better. So the mechanics of the game push you to do what's challenging to do over time, and see if you get better at it.

Charvy Narain: So both speed and accuracy are important in this game and people obviously get better by playing it. But are there generalized benefits and if so, what sort of tasks would people be getting better at?

Dr. Gazzaley: Our hypothesis was that cognitive control abilities have some underlying similarities and commonalities. And that if we improve the ability to deal with multiple tasks, we' $d$ see benefits in other cognitive control abilities that older adults are deficient in. And that's what we're able to show. We see improvement in a very different task, a working memory task, which is essentially holding an image of a face in mind for several seconds even if that face is interrupted by something else. And in a vigilance task - that is, a sustained-attention, boring, repetitive task to identify a rare target - there is also improvement. So it was encouraging to see two tasks that were not explicitly trained in the game improve in the training group, but not in two control groups.

Charvy Narain: And what is the extent of these improvements? If you start with older adults, do they begin to resemble younger people?

Dr. Gazzaley: They do. They resemble the performance of 20 year olds. And neurally they start resembling them. So we use EEG recordings as an outcome measure, preimposed. We measure prefrontal cortical activity, midline frontal theta, that's engaged and bursts right at the most challenging part of the game, a third of a second after a sign comes up when you're also driving. This measure is deficient in 60-70 year olds compared with 20 year olds before the game; then after the game it's not any different.
So we can see both neurally and behaviorally a renormalization of a lot of these patterns.

Charvy Narain: How much training does it take, and how long do the benefits last?

Dr. Gazzaley: In this study, it was 12 hours of training, 1 hour a day, 3 days a week, for a month. We tested after 6 months by having subjects play the game itself, and measuring how they did with the two tests at the same time. There was no decline in their abilities 6 months later, which was pretty shocking. Had we known that would happen, we would have done more detailed analysis to see what else remained. Some of the studies we're doing now are to try to understand how skill is retained over such long periods of time.

Charvy Narain: What sort of future work are you planning? Where do you think this technology will lead you?

Dr. Gazzaley: We have a lot of new games that target different cognitive processes. For example, one study is focused on a different type of distraction, internal distraction. It uses the principle of concentrative meditation and then integrates it with the video game dynamics of adaptivity and feedback to create an internal attention video game. We will see if that benefits older adults. We're also moving into different populations. We'd like to see if we can help patients with developmental disorders and ADD, depression, PTSD, traumatic brain injury, or dementia. It's exciting to see if there could be an impact in other populations. We're also working hard to see if we can have the adaptivity of the video game be guided by more than just performance. We think the special ingredient is the closed loop approach, in which you get better as the game gets more challenging. How about if the game doesn't just know how you're performing but knows what's going on in your brain? Maybe even looking at aspects of how your brain is functioning that are not even clearly represented in behaviors. So, the goal is to get real-time EEG recordings and feed those signals into the game mechanics. In essence, the game could diagnose deficits in how your brain processes information in real time and then guide the adaptivity and feedback to target those processes. The video game is not a magic trick, it activates a circuit and we could use other approaches to boost those effects further. 


\section{$\$_{\text {CSH }}^{\infty}$ Cold Spring Harbor Symposia SYMPOSIA On Quantitative Biology}

\section{A Conversation with Adam Gazzaley}

Cold Spring Harb Symp Quant Biol 2014 79: 275-276

Access the most recent version at doi:10.1101/sqb.2014.79.10

\section{License}

Email Alerting Receive free email alerts when new articles cite this article - sign up in Service the box at the top right corner of the article or click here. 